\title{
Antimicrobial Susceptibility Pattern of Bacterial Isolates from Surgical Site Infections in a Tertiary Care Hospital
}

\author{
Anusuya Devi Devaraju* and S. Latha Roy \\ Department of Microbiology, Dr.Chandramma Dayananda Sagar Institute of Medical \\ Education and Research, Harohalli, Ramanagara Dt, Karnataka, India \\ *Corresponding author
}

\section{Keywords}

Surgical site infections (SSI), methicillin resistant Staphylococcus aureus (MRSA)

\section{Article Info}

Received:

14 October 2021 Accepted: 04 November 2021 Available Online: 10 November 2021

\section{A B S T R A C T}

Background Surgical site infections are ranked among the most common health care associated infections. They cause significant morbidity, increased cost of care and prolonged hospital stay. A spectrum of microorganisms with varied antimicrobial susceptibility patterns have been identified as causative agents of SSI which vary with time, hospital, and with the type of surgical procedure performed. We conducted this study with an objective to assess the burden of SSI, its causative aerobic bacteria and their in vitro antibiotic susceptibility patterns. Aims \& objectives 1) To identify the aerobic bacteriological profile of isolates causing surgical site infections. 2) To determine the antibiotic sensitivity pattern of the isolates. Method: This study includes 266 clinically diagnosed cases of SSIs over a period of 8 months. Isolates were identified by conventional methods. Isolates of Staphylococcus aureus were tested for methicillin resistance by cefoxitin. Isolates of Escherichia coli and Klebsiella pneumoniae which showed resistance to cefotaxime and ceftazidime were tested for ESBL production by CLSI guidelines. Isolates of Pseudomonas aeruginosa were screened for MBL production using Imipenem disc diffusion test. Results of the 266 samples processed, 193(72.5\%) were culture positive samples which yielded 204 isolates. Staphylococcus aureus $60(29.4 \%)$ was found to be the predominant organism causing SSI followed by Pseudomonas aeruginosa 40 (19.6\%). Methicillin resistance was observed in $12(20 \%)$ of Staphylococcus aureus strains. ESBL production was observed in $20.5 \%$ of Klebsiella pneumoniae isolates and $13.2 \%$ Escherichia coli isolates. MBL production was not seen Pseudomonas aeruginosa isolates. Conclusion: The present study showed the commonest bacteria responsible for the surgical site infections like Staphylococcus aureus, Pseudomonas aeruginosa and Escherichia coli. Hence Implementation of an effective infection control programme and judicious use of antibiotic prophylaxis reduces the incidence of SSI in the hospital. 


\section{Introduction}

Surgical site infections (SSI) are the third most commonly reported nosocomial infection and they account for approximately a quarter of all nosocomial infections. Most nosocomial surgical site infections (60-80\%) occur in the incision, some involve deep soft tissue or adjacent sites. ${ }^{1}$ Several studies have demonstrated a prolonged stay of hospitalization and associated financial burdens for patients with SSI compared to non-infected patients underwent similar surgical procedures. ${ }^{2,3}$ The risk of developing SSI is affected by many factors. These include the degree of microbial contamination of the operation site indicated by wound class as clean, clean contaminated, contaminated and dirty, and also by patient age, length of surgery, pre-operative shaving of the operative site, hypothermia and co-morbidities e.g. diabetes and obesity. ${ }^{4}$

The accumulation of serous, serosanguinous or purulent material either as abscess or exuding from a muco-cutaneous surface is one of the signs of local infection. ${ }^{5}$ Sources of surgical site infections can include the patient's own normal flora or organisms present in the hospital environment. The common organisms encountered in postoperative wound infections are Staphylococcus aureus, CONS, Enterococci, Proteus, Pseudomonas, Escherichia coli and Klebsiella species. ${ }^{6,7}$ This study is being undertaken to identify the common causative organisms of surgical site infections and suggest effective antibiotics which will help to reduce morbidity, mortality and duration of stay in the hospital.

\section{Materials and Methods}

This study was carried out in the department of Microbiology, Dr. Chandramma Dayananda Sagar institute of Medical education and research, Harohalli, Ramanagara Dt, Karnataka during the period of 8 months from January2021 to August2021. Samples were collected after obtaining consent. Institutional ethics committee approval was obtained. Inclusion criteria:

All clinically diagnosed cases of surgical site infections of all age groups, irrespective of preoperative administration of antibiotics,

Those who stayed for at least seven days postoperatively were included in this study.

\section{Exclusion criteria}

Cases of anaerobic bacteria and fungal isolates were excluded from the study.

\section{Sample collection and method}

A total of 266 clinically diagnosed cases of surgical site infections admitted in various surgical wards at Dr. Chandramma Dayananda Sagar institute of Medical education and research, Harohalli, Ramanagara Dt, Karnataka were included in this study. Samples were received in laboratory either as a wound swab or as an aspirate from the infected wound. The samples were immediately cultured on bloodagar, MacConkey's agar. Bacterial isolates were identified as per standard protocol. ${ }^{8}$

\section{Antimicrobial susceptibility testing}

This was done on Mueller Hinton Agar by Kirby Bauer disc diffusion method according to the CLSI guidelines 2018. ${ }^{9}$ Bacterial isolates were tested against ampicillin/sulbactum $(10 \mu \mathrm{g} / 10 \mu \mathrm{g})$, amikacin $(30 \mu \mathrm{g}), \quad$ gentamicin $(10 \mu \mathrm{g}), \quad$ cefotaxime $(30 \mu \mathrm{g})$, ceftriaxone $(30 \mu \mathrm{g})$, ceftazidime $(30 \mu \mathrm{g}), \quad$ cotrimoxazole $\quad(1.25 / 23.75 \mu \mathrm{g})$, ciprofloxacin $(10 \mu \mathrm{g}), \quad$ Piperacillin $(100 \mu \mathrm{g})$ piperacillin-tazobactum $\quad(100 / 10 \mu \mathrm{g})$, 
cefeperazone-sulbactum $\quad(30 \mu \mathrm{g} / 10 \mu \mathrm{g})$, cefepime $(30 \mu \mathrm{g})$, Imipenem $(10 \mu \mathrm{g})$. Staphylococci were tested against Amoxy-clav $(10 \mu \mathrm{g} / 10 \mu \mathrm{g})$, amikacin $(30 \mu \mathrm{g})$, gentamicin $(10 \mu \mathrm{g})$ high-level gentamicin $(120 \mu \mathrm{g})$ for

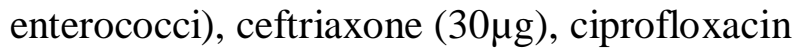
$(10 \mu \mathrm{g})$, erythromycin $(15 \mu \mathrm{g})$, clindamycin $(2 \mu \mathrm{g}), \quad$ cotrimoxazole $(1.25 / 23.75 \mu \mathrm{g})$, vancomycin $(30 \mu \mathrm{g})$, linezolid $(30 \mu \mathrm{g})$ and cefoxitin $(30 \mu \mathrm{g})$.

Test for Detection of ESBL Production in Enterobacteriaceae

Isolates which were resistant to third generation cephalosporins were tested for ESBL production by combination disk method

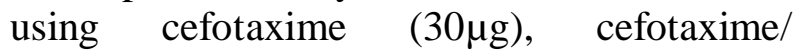
clavulanic acid $(30 \mu \mathrm{g} / 10 \mu \mathrm{g})$, and ceftazidime $(30 \mu \mathrm{g}), \quad$ ceftazidime/ clavulanic acid $(30 \mu \mathrm{g} / 10 \mu \mathrm{g})$. Plates were incubated overnight at $37^{\circ} \mathrm{C}$. Zone of inhibition of $\geq 5 \mathrm{~mm}$ around cephalosporin + clavulanate compared to cephalosporin alone confirms ESBL production. $^{9}$

\section{Test for detection of Methicillin resistance in Staphylococcus}

The test was carried out on Mueller-Hinton agar using a cefoxitin disc $(30 \mu \mathrm{g})$ and incubated at $35^{\circ} \mathrm{C}$ for $18-24 \mathrm{hrs}$. An inhibition zone diameter of $\leq 21 \mathrm{~mm}$ was reported as methicillin resistant and a diameter of $\geq 22$ $\mathrm{mm}$ was reported as methicillin sensitive strains. ${ }^{9}$

\section{Results and Discussion}

A total number of 266 samples were collected during the period of study, out of which 193(72.5\%) were culture positive samples which yielded 204 isolates. Most of the samples were obtained from individuals between the age of 40-60 yrs. Infection rate was more common in males(66.3\%) compared to females $(36.7 \%)$. The most common isolate were Staphylococcus aureus 60 (29.4\%), Pseudomonas aeruginosa 40(19.6\%), Klebsiella pneumoniae 34(16.6\%), Escherichia coli 29 (14.2\%) followed by Staphylococcus epidermidis $16 \quad(7.8 \%)$ Proteus mirabilis 11 (5.3\%), Citrobacter koseri 8 (3.9\%), Enterococcus species 4 (1.9\%), Acinetobacter species 2 (0.9\%) (Table1)

The predominant isolate Staphylococcus aureus 60 (29.4\%) showed maximum sensitivity towards vancomycin (100\%), linezolid (100\%), amikacin (96\%), Gentamycin $(89.7 \%)$ and least sensitive to ciprofloxacin $(22.2 \%)$, amoxyclav (33.3\%), ceftriaxone $(52.3 \%)$.(Table 2$)$

Among the 60(29.4\%) Staphylococcus aureus isolates, $12(20 \%)$ were found to be MRSA by cefoxitin disc diffusion test. All the MRSA strains were found to be $100 \%$ sensitive to Vancomycin and Linezolid.(Table 4)

Pseudomonas aeruginosa 40(19.6\%) was the second most common isolated organism and it was most sensitive to Imipenem $(100 \%)$, Piperacillin-tazobactum (85\%), and least sensitive to Piperacillin (14.2\%), cefepime (37.5\%), ciprofloxacin (47.5\%).

Klebsiella pneumoniae $34(16.6 \%)$ was the third most common isolated organism and it was most sensitivite to Imipenem (100\%), piperacillin-tazobactum (91.2\%) amikacin $(60 \%)$ and least sensitive to ampicillin/sulbactum (21\%), cotrimoxazole $(45.6 \%)$ and ciprofloxacin $(54.3 \%)$.

Escherichia coli29(14.2\%) was found to be maximum sensitivity towards Imipenem (100\%), piperacillin-tazobactum (94.5\%), Amikacin (64.2\%) and they were least sensitive towards ampicillin/sulbactum (21.6\%), ciprofloxacin (37.5\%) and 
cotrimoxazole (52\%).(Table 3). Among the 68 Enterobacteriaceae isolates, $23(33.8 \%)$ were ESBL producers. Highest prevalence of ESBL production was seen in Klebsiella pneumoniae 14 (20.5\%), followed by Escherichia coli 9(13.2\%). The ESBL producing strains showed maximum sensitivity towards Imipenem and piperacillin-tazobactum and $100 \%$ resistance towards third generation cephalosporins and ampicillin/sulbactum.

Surgical site infections still remains one of the most important causes of morbidity and is the most common nosocomial infection in surgically treated patients. ${ }^{2,10}$ The problem of post operative wound infection is seen in both developing and developed countries despite meticulous surgical practice and proper sterilization techniques. ${ }^{11}$

The incidence of SSI were the most common health-care associated infection accounting for $31 \%$ of all HAI among hospitalized patients. ${ }^{12}$

In our study incidence of SSI was higher in males (66.3\%) as compared to females (36.7\%). Male gender is associated with a dramatically increased risk of major infections following trauma. A study done by kumar et al., showed a similar trend $25.6 \%$ in male and $17.4 \%$ in female. ${ }^{13}$

Rate of SSI increase with the increase in age. In the present study a higher proportion of SSI was found above the age of $50 \mathrm{yrs}$. This is similar to other studies. ${ }^{14-1}$ This is due to poor immune response, underlying risk factors in old age, and reduced compliance with treatment. ${ }^{6}$ In our study, we obtained 193 culture positive samples which yielded 204 isolates from 266 suspected postoperative wound infection samples. Staphylococus aureus60 (29.4\%) was the predominant organism isolated from surgical sites followed by Pseudomonas aeruginosa 40 (19.6\%), Klebsiella pneumoniae 34 (16.6\%), Escherichia coli $29(14.2 \%)$ in the present study. Staphylococcus epidermidis 16 (7.8\%) Proteus mirabilis 11 (5.3\%), Citrobacter koseri 8 (3.9\%), Enterococcus species 4 (1.9\%), Acinetobacter species $2(0.9 \%)$ were the other organisms isolated from SSIs.

Many studies also found Staphylococcus aureus and Pseudomonas aeruginosa as the commonest isolate from the post-operative wound infection which is concordance with the present study. ${ }^{17-19}$

Table.1 Distribution of culture positive cases according to spectrum of bacterial isolates

\begin{tabular}{|c|c|c|}
\hline Sl.No. & Organisms & Total No(\%) \\
\hline $\mathbf{1}$ & Staphylococcus aureus & $60(29.4)$ \\
\hline $\mathbf{2}$ & Pseudomonas aeruginosa & $40(19.6)$ \\
\hline $\mathbf{3}$ & Klebsiella pneumoniae & $34(16.6)$ \\
\hline $\mathbf{4}$ & Escherichia coli & $29(14.2)$ \\
\hline $\mathbf{5}$ & Staphylococcus epidermidis & $16(7.8)$ \\
\hline $\mathbf{6}$ & Proteus mirabilis & $11(5.3)$ \\
\hline $\mathbf{7}$ & Citrobacter koseri & $8(3.9)$ \\
\hline $\mathbf{8}$ & Enterococcus species & $4(1.9)$ \\
\hline $\mathbf{9}$ & Acinetobacter species & $2(0.9)$ \\
\hline & Total & 204 \\
\hline
\end{tabular}


Table.2 Antibiotic sensitivity pattern of Gram positive organisms

\begin{tabular}{|c|c|c|c|c|c|c|c|c|c|c|c|c|}
\hline $\begin{array}{l}\text { Sl. } \\
\text { No. }\end{array}$ & $\begin{array}{c}\text { Organisms } \\
\text { Total-80 }\end{array}$ & $\begin{array}{c}\text { AMC } \\
(\%)\end{array}$ & $\begin{array}{c}\text { G/HLG } \\
(\%)\end{array}$ & $\begin{array}{l}\text { Ak } \\
(\%)\end{array}$ & $\begin{array}{l}\text { Ctr } \\
(\%)\end{array}$ & $\begin{array}{l}\text { Cf } \\
(\%)\end{array}$ & $\begin{array}{l}\text { Co } \\
(\%)\end{array}$ & $\begin{array}{c}\mathrm{E} \\
(\%)\end{array}$ & $\begin{array}{l}\text { CD } \\
(\%)\end{array}$ & $\begin{array}{l}\mathbf{L z} \\
(\%)\end{array}$ & $\begin{array}{l}\text { Cn } \\
(\%)\end{array}$ & $\begin{array}{l}\text { Va } \\
(\%)\end{array}$ \\
\hline 1 & $\begin{array}{c}\text { Staphylococcus } \\
\text { aureus (60) }\end{array}$ & $\begin{array}{c}20 \\
(33.3)\end{array}$ & $\begin{array}{c}54 \\
(89.7)\end{array}$ & $\begin{array}{c}58 \\
(96)\end{array}$ & $\begin{array}{c}31 \\
(52.3)\end{array}$ & $\begin{array}{c}13 \\
(22.2)\end{array}$ & $\begin{array}{c}28 \\
(46.6)\end{array}$ & $\begin{array}{c}40 \\
(66.6)\end{array}$ & $\begin{array}{c}45 \\
(75)\end{array}$ & $\begin{array}{c}60 \\
(100)\end{array}$ & $\begin{array}{c}48 \\
(80)\end{array}$ & $\begin{array}{c}60 \\
(100)\end{array}$ \\
\hline 2 & $\begin{array}{c}\text { Staphylococcus } \\
\text { epidermidis } \\
\text { (16) }\end{array}$ & $\begin{array}{c}4 \\
(25)\end{array}$ & $\begin{array}{c}8 \\
(50)\end{array}$ & $\begin{array}{c}10 \\
(62.5)\end{array}$ & $\begin{array}{c}13 \\
(81.2)\end{array}$ & $\begin{array}{c}7 \\
(43.7)\end{array}$ & $\begin{array}{c}6 \\
(37.5)\end{array}$ & $\begin{array}{c}9 \\
(56.2)\end{array}$ & $\begin{array}{c}12 \\
(75)\end{array}$ & $\begin{array}{c}16 \\
(100)\end{array}$ & $\begin{array}{c}16 \\
(100)\end{array}$ & $\begin{array}{c}16 \\
(100)\end{array}$ \\
\hline 3 & $\begin{array}{c}\text { Enterococcus } \\
\text { species (4) }\end{array}$ & $\begin{array}{c}\mathbf{0} \\
(\mathbf{0})\end{array}$ & $\begin{array}{c}2 \\
(\mathbf{5 0})\end{array}$ & $\begin{array}{c}2 \\
(50)\end{array}$ & $\begin{array}{c}2 \\
(50)\end{array}$ & $\begin{array}{c}3 \\
(60)\end{array}$ & $\begin{array}{c}2 \\
(50)\end{array}$ & $\begin{array}{c}4 \\
(100)\end{array}$ & $\begin{array}{c}4 \\
(100)\end{array}$ & $\begin{array}{c}4 \\
(100)\end{array}$ & $\begin{array}{c}4 \\
(100)\end{array}$ & $\begin{array}{c}4 \\
(100)\end{array}$ \\
\hline
\end{tabular}

Abbreviations:Amp/sul-Ampicillin/sulbactum, G-Gentamicin,HLG-high level Gentamicin, Ak-Amikacin, Ctrceftriaxone,Cf-Ciprofloxacin, Co-Cotrimoxazole, E-erythromycin, CD- clindamycin,Lz-linezolid,Cn-cefoxitin, Vavancomycin.

Table.3 Antibiotic sensitivity pattern of Gram negative organisms

\begin{tabular}{|c|c|c|c|c|c|c|c|c|c|c|c|c|c|c|}
\hline $\begin{array}{l}\text { Sl. } \\
\text { No. }\end{array}$ & $\begin{array}{c}\text { Isolates } \\
\text { Total no isolates } \\
\text { (124) }\end{array}$ & $\begin{array}{c}\text { Amp/ } \\
\text { sul } \\
(\%)\end{array}$ & $\begin{array}{c}\text { G } \\
(\%)\end{array}$ & $\begin{array}{l}\text { Ak } \\
(\%)\end{array}$ & $\begin{array}{l}\text { cf } \\
(\%)\end{array}$ & $\begin{array}{l}\text { Co } \\
(\%)\end{array}$ & $\begin{array}{c}\text { Cpz/sul } \\
(\%)\end{array}$ & $\begin{array}{c}\mathrm{Ca} \\
(\%)\end{array}$ & $\begin{array}{l}\mathrm{Ce} \\
(\%)\end{array}$ & $\begin{array}{l}\text { Ctr } \\
(\%)\end{array}$ & $\begin{array}{c}\text { Cpm } \\
(\%)\end{array}$ & $\begin{array}{l}\text { Pc } \\
(\%)\end{array}$ & $\begin{array}{l}\mathbf{P t} \\
(\%)\end{array}$ & $\underset{(\%)}{I}$ \\
\hline $\mathbf{1}$ & $\begin{array}{c}\text { Pseudomonas } \\
\text { aeruginosa }(40)\end{array}$ & $\begin{array}{c}11 \\
(27.5)\end{array}$ & $\begin{array}{c}22 \\
(55)\end{array}$ & $\begin{array}{c}30 \\
(75)\end{array}$ & $\begin{array}{c}19 \\
(47.5)\end{array}$ & $\begin{array}{c}0 \\
(100)\end{array}$ & $\begin{array}{c}34 \\
(85 \%)\end{array}$ & $\begin{array}{c}33 \\
(82.5)\end{array}$ & $\begin{array}{c}32 \\
(80)\end{array}$ & $\begin{array}{c}34 \\
(85 \%)\end{array}$ & $\begin{array}{c}15 \\
(37.5)\end{array}$ & $\begin{array}{c}6 \\
(14 . \\
2)\end{array}$ & $\begin{array}{c}34 \\
(85 \%)\end{array}$ & $\begin{array}{c}40 \\
(100)\end{array}$ \\
\hline 2 & $\begin{array}{c}\text { Klebsiellapneum } \\
\text { oniae (34) }\end{array}$ & $\begin{array}{c}7 \\
(20.5)\end{array}$ & $\begin{array}{c}19 \\
(55)\end{array}$ & $\begin{array}{c}21 \\
(60)\end{array}$ & $\begin{array}{c}19 \\
(54.3)\end{array}$ & $\begin{array}{c}15 \\
(45.6)\end{array}$ & $\begin{array}{c}23 \\
(67.6)\end{array}$ & $\begin{array}{c}20 \\
(58)\end{array}$ & $\begin{array}{c}21 \\
(61.7)\end{array}$ & $\begin{array}{c}20 \\
(58)\end{array}$ & $\begin{array}{c}25 \\
(73.5)\end{array}$ & $\begin{array}{c}11 \\
(32 . \\
5)\end{array}$ & $\begin{array}{c}31 \\
(91.2)\end{array}$ & $\begin{array}{c}34 \\
(100)\end{array}$ \\
\hline 3 & $\begin{array}{c}\text { Escherichia coli } \\
\text { (29) }\end{array}$ & $\begin{array}{c}12 \\
(21.6)\end{array}$ & $\begin{array}{c}16 \\
(55.6)\end{array}$ & $\begin{array}{c}19 \\
(64.5)\end{array}$ & $\begin{array}{c}11 \\
(37.5)\end{array}$ & $\begin{array}{c}15 \\
(52)\end{array}$ & $\begin{array}{c}53 \\
(92.9)\end{array}$ & $\begin{array}{c}20 \\
(68.9)\end{array}$ & $\begin{array}{c}21 \\
(72.4)\end{array}$ & $\begin{array}{c}22 \\
(75.8)\end{array}$ & $\begin{array}{c}25 \\
(86.2)\end{array}$ & $\begin{array}{c}14 \\
(48)\end{array}$ & $\begin{array}{c}27 \\
(94.5)\end{array}$ & $\begin{array}{c}29 \\
(100)\end{array}$ \\
\hline 4 & $\begin{array}{l}\text { Proteus mirabilis } \\
\text { (11) }\end{array}$ & $\begin{array}{c}3 \\
(27.2)\end{array}$ & $\begin{array}{c}5 \\
(45.4)\end{array}$ & $\begin{array}{c}6 \\
(33.3)\end{array}$ & $\begin{array}{c}4 \\
(54.5)\end{array}$ & $\begin{array}{c}4 \\
(54.5)\end{array}$ & $\begin{array}{c}7 \\
(63.6)\end{array}$ & $\begin{array}{c}11 \\
(100)\end{array}$ & $\begin{array}{c}11 \\
(100)\end{array}$ & $\begin{array}{c}11 \\
(100)\end{array}$ & $\begin{array}{c}11 \\
(100)\end{array}$ & $\begin{array}{c}4 \\
(54 . \\
5)\end{array}$ & $\begin{array}{c}11 \\
(100)\end{array}$ & $\begin{array}{c}11 \\
(100)\end{array}$ \\
\hline 5 & $\begin{array}{c}\text { Citrobacter } \\
\text { koseri }(8)\end{array}$ & $\begin{array}{c}2 \\
(25)\end{array}$ & $\begin{array}{c}4 \\
(50)\end{array}$ & $\begin{array}{c}5 \\
(62.5)\end{array}$ & $\begin{array}{c}4 \\
(50)\end{array}$ & $\begin{array}{c}4 \\
(\mathbf{5 0})\end{array}$ & $\begin{array}{c}6 \\
(75)\end{array}$ & $\begin{array}{c}8 \\
(\mathbf{1 0 0})\end{array}$ & $\begin{array}{c}8 \\
(100)\end{array}$ & $\begin{array}{c}8 \\
(100)\end{array}$ & $\begin{array}{c}8 \\
(100)\end{array}$ & $\begin{array}{c}4 \\
(50)\end{array}$ & $\begin{array}{c}8 \\
(100)\end{array}$ & $\begin{array}{c}8 \\
(100)\end{array}$ \\
\hline
\end{tabular}

Abbreviations:Amp/sul-Ampicillin/sulbactum, G-Gentamicin, Ak-Amikacin,cf-ciprofloxacin, Co-Cotrimoxazole, Cpz/sul- cefeperazone-sulbactum, Ca-Ceftazidime, Ce-Cefotaxime,Ctr-ceftriaxone,cpmcefepime,PtPiperacillin/tazobactam, I-Imipenem,

Table.4 Antibiotic sensitivity pattern of MRSA

\begin{tabular}{|c|c|c|c|c|c|c|c|c|c|c|c|c|}
\hline $\begin{array}{l}\text { SI. } \\
\text { No. }\end{array}$ & Organisms & $\begin{array}{c}\text { AMC } \\
(\%)\end{array}$ & $\begin{array}{c}\mathbf{G} \\
(\%)\end{array}$ & $\begin{array}{c}\text { Ak } \\
(\%)\end{array}$ & $\begin{array}{l}\text { Ctr } \\
(\%)\end{array}$ & $\begin{array}{c}\text { Cf } \\
(\%)\end{array}$ & $\begin{array}{c}\text { Co } \\
(\%)\end{array}$ & $\begin{array}{c}\mathbf{E} \\
(\%)\end{array}$ & CD & $\begin{array}{c}\mathbf{L z} \\
(\%)\end{array}$ & $\begin{array}{l}\mathrm{Cn} \\
(\%)\end{array}$ & $\begin{array}{l}\text { Va } \\
(\%)\end{array}$ \\
\hline 1 & $M$ & $\begin{array}{c}\mathbf{0} \\
(\mathbf{0})\end{array}$ & $\begin{array}{c}7 \\
(58.3)\end{array}$ & $\begin{array}{c}9 \\
(75)\end{array}$ & $\begin{array}{c}10 \\
(83.3)\end{array}$ & $\begin{array}{c}6 \\
(50)\end{array}$ & $\begin{array}{c}\mathbf{0} \\
(\mathbf{0})\end{array}$ & $\begin{array}{c}10 \\
(\mathbf{8 3 . 3})\end{array}$ & $\begin{array}{c}11 \\
(91.6)\end{array}$ & $\begin{array}{c}12 \\
(100)\end{array}$ & $\begin{array}{c}\mathbf{0} \\
(\mathbf{0})\end{array}$ & $\begin{array}{c}12 \\
(100)\end{array}$ \\
\hline
\end{tabular}

Abbreviations:Amp/sul-Ampicillin/sulbactum, G-Gentamicin, Ak-Amikacin, Ctr-ceftriaxone,Cf-Ciprofloxacin, CoCotrimoxazole, E-erythromycin, CD- clindamycin,Lz-linezolid,Cn-cefoxitin, Va-vancomycin.

Staphylococcus aureus $60(29.4 \%)$ was the most common Gram positive bacteria followed by Staphylococcus epidermidis 16 (7.8\%) and Enterococcus spp 4 (1.9\%) isolated in SSI. $12(20 \%)$ strains were of
MRSA. All the MRSA strains are 100\% sensitive to Vancomycin and Linezolid.

Among Gram negative isolates, Pseudomonas aeruginosa $40(19.6 \%)$ was the most common 
organism isolated in SSI followed by Klebsiella pneumoniae 34 (16.6\%), Escherichia coli 29 (14.2\%). It has high sensitivity towards Imipenem(100\%), Piperacillin-tazobactum (85\%).

In the present study members of Enterobacteriaceae family showed high sensitivity to Imipenem, Piperacillintazobactum and amikacin.

In the present study observed $13.2 \%$ of the Escherichia coli and $20.5 \%$ of Klebsiella pneumoniae to be ESBL producers which is comparable with other studies. ${ }^{20,21}$

There is a gradual increase in the emergence of antibiotic resistant organisms in surgical patients. The majority of the Gram-negative isolates are sensitive to Imipenem and Piperacillin-tazobactum while Gram positive isolates being sensitive to vancomycin and Linezolidand partly to fluoroquinolones. These findings evident the need for effective infection control and rational use of antimicrobial agents leading to minimize infection rate and emergence of drug resistance. The surgical site infections are the commonest nosocomial infection in hospital acquired infection. The present study showed the commonest bacteria responsible for the surgical site infections like Staphylococcus aureus, Pseudomonas aeruginosa and Escherichia coli. Hence the study concludes that strict major needs to be taken to avoid such surgical site infections. Also the treatment of this may leads to the antibiotic resistance. The data in the present study may be useful in choosing the effective therapy against the isolates from surgical site infections.

\section{Ethical approval}

The study was approved by the Institutional Ethics Committee

\section{References}

1. Wong E S. Surgical site infections. In: Mayhall CG, editor. Hospital epidemiology and infection control. 1st ed.U.S.A: Williams and Wilkins; 1996.p.154-74.

2. Urban J A. Cost analysis of surgical site infections. Surg Infect(Larchmt)2006;7(suppl 1):S19-22.

3. Reichman D E, Greenberg J A. Reducing Surgical Site Infections: A Review. Rev ObstetGynecol2009;2:212-21.

4. Mangram A J, Horan T C, Pearson M L, Silver L C, Jarvis W R. Guidelines for prevention of surgical site infection. Infect Control Hosp Epidemiol 1999;20: 250278.

5. Koneman E W. Allen S D, Janda W M, Schreckenberger P C, Winn W C Jr, editors. Color Atlas and Textbook of Diagnostic Microbiology. 5th Ed. Lipincott-Raven Publishers: Philadelhia: Pa; 1997.

6. Anderson D J, Sexton D J, Kanafani Z A, Auten G, Kaye K S. Severe surgical site infection in community hospitals: epidemiology, key procedures and the changing prevalence of methicillin resistant Staphylococcus aureus. InfectControl Hosp Epidemiol 2007;28:1047-1053.

7. Cantlon C A, Stemper M E. Schwan W R, Hoffman M A, Qutaishat S S. Significant pathogens isolated from surgical site infections at a community hospital in the Midwest. Am J Infect Control 2006; 34: 526-529.

8. Koneman E W. Allen S D, Janda W M, Schreckenberger P C, Winn W C Jr, editors. Color Atlas and Textbook of Diagnostic Microbiology. 5th Ed. Lipincott-RavenPublishers: Philadelhia: $\mathrm{Pa} ; 1997$.

9. Clinical and Laboratory Standards Institute (CLSI). Performance standards 
for antimicrobial susceptibility testing. $17^{\text {th }}$ informational supplement: 2018.p. M100-S17.

10. Desa L A, Sathe M J. Factors influencing wound infection (a prospective study of 280 cases). JPostgrad Med1984;30:231-6.

11. Mundhada A S, Tenpe S. A study of organisms causing surgical site infections and their antimicrobial susceptibility in a tertiary care Government Hospital. Indian J PatholMicrobiol2015;58:195-200.

12. CDC. CDC/NHSN protocol clarifications, 2013. Available at:http:/ /www. cdc.gov/nhsn/pdf/ pscmanual / protocol clarification.pdf.

13. Ashok Kumar Singh, Upendra Nath. Surgical Site Infection in Abdominal Surgeries in a Tertiary Centre. 2016;6(3). ISSN -2249-555X | IF:3.919 | IC Value : 74.50Resea)

14. National Academy of Science/ National Research Council. Post operative wound infections: Influence of ultraviolet irradiation of the operating room and of various other factor.Ann Surg1964;160(supp2):1-132.

15. Xue D Q, Qian C, Yang L, Wang X F. Risk factors for surgical site infections after breast surgery: A systematic review and meta-analysis. Eur J SurgOncol2012;38:375-81.

16. Ashby E, Davies M J, Wilson A P, Haddad F S. Age, ASA and BMI as risk factors for surgical site infection measured using ASEPSIS in trauma and orthopaedic surgery. J Bone Joint Surg Br 2012;94 (SUPP 4):58.

17. Mahesh C B, Shivakumar S, Suresh B S, Chidanand S P, Vishwanath Y. A prospective study of surgical site infections in a teaching hospital. J Clin Diagn Res2010;4:3114-9.

18. Malik S, Gupta A, Singh K P, Agarwal J, Singh M. Antibiogram of Aerobic Bacterial Isolates from Post-operative Wound Infections at a Tertiary Care Hospital in India. J Infect Dis Antimicrob Agents 2011;28:45-52.

19. Mangram A J, Horan T C, Pearson M L, Silver L C, Jarvis W R. Guideline for prevention of surgical site infection, 1999. Hospital Infection Control Practices Advisory Committee. Infect Control HospEpidemiol2017;20:250-78.

20. Aniruddha S. Mundhada, Sunita Tenpe. A study of organisms causing surgical site infections and their antimicrobial susceptibility in a tertiary care Government Hospital. Eur JSurgOncol2012;38:375-81.

21. Mawalla B, Mshana S E, Chalya P L, Imirzalioglu C, Mahalu W. Predictors of surgical site infections among patients undergoing major surgery at Bugando Medical Centre in North western Tanzania. BMC Surg2011;11(1):21.

\section{How to cite this article:}

Anusuya Devi Devaraju and Latha Roy, S. 2021. Antimicrobial Susceptibility Pattern of Bacterial Isolates from Surgical Site Infections in A Tertiary Care Hospital. Int.J.Curr.Microbiol.App.Sci. 10(11): 369-375. doi: https://doi.org/10.20546/ijcmas.2021.1011.042 